\title{
AUTO-EXAME DE MAMA: CONHECIMENTO DE USUÁRIAS ATENDIDAS NO AMBULATÓRIO DE UMA MATERNIDADE ESCOLA
}

\author{
Rejane Marie Barbosa Davim ${ }^{1}$ \\ Gilson de Vasconcelos Torres ${ }^{2}$ \\ Maria Lúcia Nunes Cabral ${ }^{3}$ \\ Vilma Maria de Lima $^{4}$ \\ Maria Anakarine de Souza ${ }^{5}$
}

Davim RMB, Torres GV, Cabral MLN, Lima VM, Souza MA. Auto-exame de mama: conhecimento de usuárias atendidas no ambulatório de uma maternidade escola. Rev Latino-am Enfermagem 2003 janeiro-fevereiro; 11(1):21-7.

Estudo descritivo desenvolvido no ambulatório de uma Maternidade Escola em, Natal/RN. Objetivou-se identificar o conhecimento e as alterações encontradas no auto-exame de mama e descrever como as mulheres se auto-examinam. A população foi constituída por mulheres referenciadas de outros serviços de saúde, e a amostra constou de 109 mulheres que tinham entre 15 a 83 anos de idade. O instrumento para a coleta de dados foi um formulário de entrevista contendo dados de identificação e acerca do auto-exame de mama. A partir dos dados coletados, identificamos que, das 109 participantes do estudo, 36 (33\%) estavam entre 15 a 39 anos de idade; 58 (53\%) entre 40 a 58 anos, e 15 (14\%), na faixa entre 60 a 83 anos. $75 \%$ referem se auto-examinarem e, ao detectarem alguma alteração nas mamas durante o auto-exame, procuraram o médico para o tratamento correto.

DESCRITORES: auto-exame, conhecimento

\section{BREAST SELF-EXAMINATION: THE KNOWLEDGE OF USERS ASSISTED IN THE OUTPATIENT UNIT OF A UNIVERSITY MATERNITY HOSPITAL}

This is a descriptive study developed in the outpatient unit of a university maternity hospital in the city of Natal/RN. It aimed at identifying the knowledge about and alterations found during breast self-examination as well as at describing how women examine themselves. The population was formed by patients sent by other health services and the sample consisted of 109 women with age varying from 15 to 83 years. In order to collect data, the authors used a form containing identification information as well as about breast self-examination. Based on the collected data, they identified that among the 109 participants, the age of 36 (33\%) of them varied from 15 to 39 years; of 58 (53\%) of them from 40 to 58 years and of 15 (14\%) of them from 60 to 83 years. $75 \%$ reported that they examined themselves and that they attended a health care service for correct treatment in case any alterations were detected during self-examination.

DESCRIPTORS: self-examination, knowledge

AUTO-EXAMEN DE LA MAMA: CONOCIMIENTO DE LAS USUARIAS ATENDIDAS EN EL AMBULATORIO DE UN HOSPITAL UNIVERSITARIO

Estudio descriptivo desarrollado en el Servicio de Consulta Externa de un Hospital Universitario de la ciudad de Natal/ RN. Tuvo como objetivo identificar el conocimiento y las alteraciones encontradas en el auto-examen de mama y describir con esas mujeres si se autoexaminan. La población estuvo constituida por mujeres remitidas de otros servicios de salud y la muestra constó de 109 mujeres que tenían entre de 15 y 83 años de edad. El instrumento utilizado para la recolección de datos fue un formulario de entrevista contiendo datos de la identificación acerca de el auto-examen de mama. A partir de los datos recolectados identificamos que de las 109 participantes del estudio, 36 (33\%) estaban entre 15 y 39 años de la edad; 58 (53\%) entre 40 y 58 años y 15 (14\%) entre 60 y 83 años. 75\% informaron que se autoexaminan y al detectar alguna alteración en las mamas durante el auto-examen, procuran el médico para el tratamiento correcto.

DESCRIPTORES: autoexamen, conocimiento

\footnotetext{
${ }^{1}$ Enfermeira Obstétrica, Professor Adjunto do Departamento de Enfermagem/CCS/UFRN, Mestre em Enfermagem de Saúde Pública/CCS/UFPB, Doutoranda em Ciências da Saúde/UFRN, Coordenadora do Curso de Especialização em Enfermagem Obstétrica: Habilidades Midwifery para uma Maternidade Segura/UFRN, e-mail: rejanemb@uol.com.br; ${ }^{2}$ Enfermeiro Doutor, Professor Adjunto do Departamento de Enfermagem/CCS/ UFRN, Coordenador da Base de Pesquisa Enfermagem Clínica, ${ }^{3}$ Enfermeira, Professor Adjunto do Departamento de Enfermagem/CCS/UFRN, Mestre em Enfermagem/CCS/UFRN; ${ }^{4}$ Enfermeira, Professor Adjunto do Departamento de Enfermagem/CCS/UFRN, Mestre em Ciências da Enfermagem/UNIRIO; ${ }^{5}$ Enfermeira do PSF no município de São Pedro/RN
} 
INTRODUÇÃO

Por meio da nossa vivência no atendimento à mulher, em instituição universitária, pudemos observar que as mesmas têm pouco conhecimento a respeito das possíveis causas e do comprometimento de suas vidas em decorrência das doenças. Sabemos que, na área da saúde, os avanços tecnocientíficos mostraram-se relevantes neste último século, quando foram descobertos tratamentos para várias doenças, prolongando, assim, muitas vidas. Dentre essas doenças, o câncer de mama é tema de constantes pesquisas e discussões na área da saúde, merecendo atenção especial de pesquisadores voltados para essa área, devido ao número crescente de casos e óbitos.

$\mathrm{Na}$ mulher, o câncer de mama afeta profundamente suas dimensões biopsicosocioespirituais, tendo-se em conta ser uma doença que, a longo ou médio prazo, resulta em mutilação da mama, podendo levar a mulher à morte e, em conseqüência, resultar uma gama de sentimentos negativos, tais como, ansiedade, choque, desespero, depressão e medo.

Para isso, a necessidade de programas de prevenção e detecção precoce do câncer de mama tem, como maior justificativa, proporcionar maiores chances de cura e ou sobrevida mais longa à mulher. Sabemos que, na rede básica de saúde, o atendimento deixa muito a desejar, além de existirem preconceitos e timidez de algumas mulheres no que se refere à realização do autoexame de mama. Porém, nos últimos anos, tem-se discutido bastante sobre a necessidade de se ensinar o auto-exame de mama, visto ser um dos métodos importantes na detecção precoce do câncer de mama, o qual possibilita à mulher, ao se examinar, conhecer as alterações mamárias e detectar precocemente o nódulo mamário. Quando realizado de forma sistemática, o autoexame de mama reveste-se de magna importância, não só pelos objetivos a que se propõe, mas também por se revelar como o menos oneroso e o mais prático de todos os exames.

Como a mulher ainda oferece resistência e dificuldade na sua realização, é necessário que essa prática seja estimulada constantemente e orientada por profissionais da área da saúde, inclusive pela enfermeira, fazendo com que conheça melhor o seu corpo e crie hábitos de se auto-examinar, visto ser este um dos métodos essenciais na detecção precoce do câncer de mama.
Diante dessas considerações, torna-se evidente a conscientização das pessoas em relação ao problema do câncer de mama. Para que isso ocorra, deve haver a participação efetiva dos profissionais da área, assim como dos usuários dos serviços de saúde e de lideranças comunitárias, visando ao ensino teórico-prático do autoexame de mama e outros assuntos de interesse geral da mulher. Sabemos que, por meio de um sistema de saúde organizado e hierarquizado em níveis de complexidade no seu atendimento, torna-se um grande fator para o bom desenvolvimento de qualquer ação de saúde.

Sendo assim, é oportuno pensar em estratégias que possam intervir nos processos que afetam particularmente a saúde da mulher trabalhadora, com seus riscos invisíveis. Para que isso se concretize, é necessária a educação em saúde, mediante campanhas educativas que envolvam o auto-exame de mama, a necessidade de prevenção ginecológica anual e o desenvolvimento de ações educativas que envolvem a saúde da mulher.

Considerando a saúde da mulher como um problema de saúde pública, a motivação em realizarmos este estudo surgiu a partir da nossa observação diária e de nossa experiência profissional, no convívio com a clientela feminina do ambulatório da Maternidade Escola Januário Cicco (MEJC), da Universidade Federal do Rio Grande do Norte (UFRN), em Natal/RN. Observamos, ao longo do tempo, que os problemas por elas vivenciados quase sempre são referentes a fatores de ordem social, econômica e cultural. Dentre esses fatores, detectamos a falta de conhecimento e a intimidade com o próprio corpo, associados ao medo de tocá-lo e sentí-lo sem culpa, medo de procurar o profissional de saúde e detectar qualquer mudança em seu organismo, medo de barreiras ao acesso nos serviços, de maltratos e negligências pelos profissionais, implicando, de certa forma, problemas referentes à sua saúde. Assim, diante de toda a problemática que envolve a saúde da mulher, este estudo tem como objetivos: identificar o conhecimento do autoexame de mama nas mulheres atendidas no ambulatório da MEJC em Natal-RN; descrever como essas mulheres realizam o auto-exame de mama e identificar quais as alterações/sintomas que são encontradas por essas mulheres durante o auto-exame.

\section{FUNDAMENTAÇÃO TEÓRICA}

O câncer de mama é uma doença progressiva, 
representando, em sua trajetória, diferentes situações de ameaça aos seus portadores, como aquelas relacionadas à integridade psicossocial, à incerteza do sucesso no tratamento, à possibilidade de recorrência, à morte, entre outros $^{(1)}$.

Por seu alto índice de mortalidade entre as mulheres e não existindo uma forma de evitar o seu aparecimento, o que melhor se pode obter é o controle de sua evolução por meio da prática sistemática do autoexame de mama e atenção, quanto aos fatores de risco.

Apesar de sua etiologia desconhecida, pesquisas nesse campo têm tentado identificar grupos ou subgrupos de mulheres com maior probabilidade de apresentar a doença. Assim, o aumento do risco para determinado fator é indicado pela freqüência em que a doença aparece entre mulheres que apresentam o fator em questão, dividido pela freqüência da doença naquelas sem o fator, que é o risco relativo ${ }^{(2)}$

Para isso, o estudo dos fatores de risco tem seguido duas linhas: clínico-epidemiológico e alterações histológicas do tecido mamário. No que se refere à primeira linha, são vários os fatores relacionados ao aumento do risco de câncer de mama: a história familiar, principalmente em familiares de primeiro grau (mãe ou irmã) que apresentaram a doença ainda jovens (abaixo de 40 anos de idade), é um importante fator de risco. Esse risco é aumentado em $50 \%$ para uma mulher jovem, cuja mãe ou irmã apresentaram a doença bilateralmente na prémenopausa. Nesses casos, existe a hipótese de que a doença apresente um caráter familiar genético e hereditário. A menarca precoce (abaixo de 12 anos) também tem sido considerada um fator de risco, porém sua ligação é fraca e apresenta um risco relativo de, aproximadamente, 1,2. A primiparidade idosa (após 30 anos) parece elevar o risco relativo do câncer de mama em duas vezes, se comparado a mulheres que tiveram o primeiro filho antes dos 20 anos de idade. Aquelas com o primeiro filho após os 35 anos, apresentam um risco discretamente superior ao das multíparas ${ }^{(2-8)}$.

Menopausa tardia (após 55 anos) representa um fator de risco importante. As mulheres nesse grupo têm o risco em dobro, relacionado àquelas com menopausa antes dos 45 anos; já a ooforectomia bilateral, antes dos 35 anos, reduz o risco para 1/3. Quanto ao uso de anovulatório oral por mais de 4 anos, parece aumentar o risco do câncer de mama, entretanto, este fator ainda é polêmico, visto que vários outros estudos não mostraram nenhuma relação. Outros fatores como lactação, ingestão de álcool, dieta rica em ácidos graxos saturados e terapia de reposição hormonal prolongada, exposição à radiação ionizante, situações emocionais de estresse são ainda mais questionáveis, não apresentando, até o momento, nenhuma relação conclusiva com o câncer de mama ${ }^{(2,4,6)}$.

Relacionando-se à segunda linha dos fatores de risco, ou seja, às alterações histológicas, o aumento no risco para o câncer de mama estaria direcionado com hiperplasias atípicas. Nesses casos, o risco relativo é de 4,3 , aproximadamente, passando para 10 , se houver associação com história familiar de câncer de mama ${ }^{(2)}$.

Apesar do grande número de pesquisas já realizadas sobre câncer, seu controle é reduzido, e, nos programas de saúde existentes, dificilmente tem êxito a redução de suas taxas de mortalidade. Dessa forma, é necessário um amplo esforço na óptica da saúde pública, para que as pesquisas sejam direcionadas na identificação dos fatores de risco e no mecanismo de ação do câncer ${ }^{(9)}$.

Estudos revelam que a redução da gordura endógena e do peso corporal, especialmente na menopausa, uma dieta rica em vitamina A e a ocorrência da primeira gestação antes dos 30 anos de idade, podem diminuir o risco de desenvolvimento do câncer de mama, já que não existe, até o momento, uma maneira comprovada de se fazer sua prevenção ${ }^{(10)}$. Assim, o diagnóstico precoce é a única proteção efetiva para o câncer de mama, existindo, para isso, três estratégias na sua detecção, que são o auto-exame de mama, o exame clínico da mama e a mamografia.

O auto-exame de mama, quando a mulher examina suas próprias mamas, exerce função importante, com possibilidade de promover o diagnóstico precoce e a cura. É de fácil execução, podendo ser realizado por mulheres pertencentes a qualquer segmento sociocultural da população. Dentre suas inúmeras vantagens, destacamse a detecção de tumorações pequenas, ainda confinadas à glândula mamária; é um método conveniente, útil, proveitoso, vantajoso e oportuno; pode ser repetido à vontade; não tem custo financeiro; é de fácil execução e sua precisão aumenta com a prática. O objetivo fundamental do auto-exame de mama é fazer com que a mulher conheça detalhadamente suas mamas, o que facilita a percepção de quaisquer alterações tais como pequenos nódulos nas mamas e axilas, saída de secreções pelos mamilos, mudança na cor da pele, retrações, entre outras, promovendo o diagnóstico precoce, 
com grandes perspectivas de cura em ritmo promissor, quando os tumores são pequenos, delimitados e localizados ainda no próprio tecido glandular mamário ${ }^{(11-}$ 13)

Apesar de o auto-exame ser recomendado indistintamente a todas as mulheres, a partir dos 21 anos de idade, entre o $7^{\circ}$ e $10^{\circ}$ dia do ciclo menstrual, quando as mamas se apresentam mais flácidas e indolores, a sua realização é obrigatória em determinados grupos de mulheres, a saber: aquelas com mais de 50 anos de idade; também cuja mãe ou irmãs tiveram ou têm câncer de mama; as que só tiveram o primeiro filho após os 30 anos de idade; nulíparas; menarca precoce e menopausa tardia; uso prolongado de hormônios estrogênicos e as que já tiveram câncer de mama. Para aquelas que não menstruam (menopausadas e histerectomizadas ou as que estão amamentando) deve-se escolher um dia no mês e realizar o auto-exame, sempre com intervalo de 30 dias ${ }^{(11-13)}$.

Ficou provado, em estudos, que mulheres, ao praticarem o auto-exame mais freqüentemente, tornam mais fácil notar qualquer modificação nas mamas, de um mês para o outro, como também apresentam tumores pequenos e um estágio clínico mais favorável da doença do que aquelas que nunca realizam essa prática. Assim, se o câncer de mama for diagnosticado em estágios iniciais, a mulher tem uma sobrevida bem melhor. Estudos na Inglaterra demonstram sobrevida em cinco anos, de $84 \%$, se o diagnóstico for realizado no estadiamento I, caindo para $18 \%$, se o estadiamento for IV. Porém o autoexame de mama parece ter uma sensibilidade baixa, possibilitando muitos falsos negativos e uma especificidade também baixa, incorrendo em falsos positivos, especialmente em mulheres mais jovens. Não há, entretanto, um consenso sobre sua melhor técnica, e as mulheres que o realizam, não seguem corretamente o que Ihes foi ensinado, além de muitas terem dificuldades em aderir a um procedimento ritualizado e com regras muito rígidas. Vale dizer que, se o auto-exame de mama não demonstrou ser eficaz para diminuir a mortalidade por câncer de mama, em ensaios clínicos, não significa afirmar que não há nada a fazer nessa área ${ }^{(11-14)}$.

As que nunca praticam o auto-exame geralmente apresentam tumores significativamente maiores do que aquelas que o praticam mensalmente ou menos que uma vez por mês. Assim, existe uma relação significante entre o tamanho do tumor primário e a presença de linfonodos axilares metastáticos, e a percentagem desses linfonodos, diagnosticados histologicamente, é menor em mulheres que praticam o auto-exame freqüentemente, aumentando, por conseguinte, o número de mulheres tratadas antes do aparecimento dessas metástases. É imprescindível, portanto, que a mulher, ao detectar qualquer alteração em sua mama, procure o médico para avaliação, aumentando, destarte, a possibilidade de cura ${ }^{(11-13)}$.

Assim, a educação em saúde, mediante campanhas educativas e orientações ambulatoriais que envolvem o auto-exame de mama, a necessidade de prevenção ginecológica anual e o desenvolvimento de ações preventivas, como a não exposição aos fatores de risco, são indispensáveis para o controle dessa patologia.

A partir de dados em pesquisas, detectou-se que $80 \%$ dos tumores de mama são descobertos pela mulher ao se palpar incidentalmente. Com essa ocorrência, eles já apresentam um tamanho grande, dificultando o seu tratamento. É válido, então, ressaltar que um tumor maligno na mama, em estágio avançado, é freqüente entre as mulheres, impossibilitando, dessa forma, o controle de sua evolução ${ }^{(11)}$.

Esse controle na evolução da doença também pode ser prejudicado pela descoberta de um nódulo mamário pela mulher, causando diversos sentimentos negativos, como é o caso da tristeza, medo e desespero. Esses sentimentos fazem com que a mulher protele, muitas vezes, a consulta médica, pelo pavor da confirmação diagnóstica. Dados do Ministério da Saúde para 1998, corroboram esse pensamento, enfatizando que $50 \%$ dos casos de câncer de mama são detectados no estágio avançado, favorecendo a alta mortalidade na população feminina ${ }^{(15)}$.

Assim, os exames periódicos da mama, realizados com freqüência, ajudam a mulher a se familiarizar com a forma, o tamanho, o aspecto da pele e do mamilo, tornando mais fácil a detecção de alguma anormalidade, possibilitando, então, um bom prognóstico $^{(16)}$.

\section{PROCEDIMENTOS METODOLÓGICOS}

Este estudo é do tipo descritivo e exploratório, desenvolvido no ambulatório da MEJC junto ao Programa Saúde da Mulher. A escolha dessa instituição deveu-se ao fato de ela ser referência do Estado do Rio Grande do Norte, campo de aulas práticas para alunos na área de saúde da UFRN, como também campo de prática 
acadêmica dos pesquisadores.

A MEJC é uma empresa hospitalar prestadora de serviços a uma comunidade carente atendida pelo Sistema Único de Saúde (SUS). Nas últimas décadas, houve um maior desenvolvimento nessa prestação de serviço, para melhor atendimento às necessidades médicas em ginecologia, obstetrícia e neonatologia.

As atividades desenvolvidas no seu plexo são de natureza propedêutica, terapêutica, preventiva e curativa, atingindo o campo ambulatorial com 16 consultórios de atendimento por meio de referências de Unidades Básicas de Saúde ou de outras instituições do Estado; oferece assistência ao parto normal e ao alojamento conjunto com vistas na humanização, objetivando servir com melhor qualidade; cirurgias obstétricas e ginecológicas; assistência à gestação, parto e recém-nascidos de alto risco; serviço de monitoragem, ultra-sonografia e reprodução humana. É hospital Amigo da Criança desde 1994, promovendo, protegendo e apoiando o aleitamento materno, mediante a adoção dos Dez Passos para Incentivo ao Aleitamento Materno e ao Método MãeCanguru.

No que se refere aos aspectos éticos, o primeiro passo para a pesquisa com sua inserção no serviço, deuse através de um ofício encaminhado à direção da instituição, solicitando autorização para a realização do estudo. Após a devida autorização, o passo seguinte foi a elaboração de um formulário com um roteiro de entrevista tendo questões objetivas e subjetivas que abordavam o conhecimento das mulheres sobre o auto-exame de mama, a detecção de alterações nas mamas por essas mulheres ao se auto-examinarem e o conhecimento sobre medidas preventivas quanto ao auto-exame. Determinamos como critério de inclusão no estudo, as mulheres que foram referenciadas de Unidades Básicas de Saúde ao ambulatório da MEJC, durante os meses de fevereiro, março e abril de 2000 , totalizando 546 encaminhamentos. A amostra constou de $20 \%$ dessa população, ou seja, 109 mulheres.

A participação das mulheres na pesquisa deu-se nos bancos de espera do ambulatório, após abordagem dos pesquisadores, momento em que lhes foi explicado os objetivos e importância do estudo, sendo a participação espontânea, com garantia de anonimato diante dos achados. Tais esclarecimentos foram pautados no consentimento livre e esclarecido, observando-se o previsto na Resolução 196/96 do Conselho Nacional de Saúde, que trata de pesquisas envolvendo seres humanos ${ }^{(17)}$.

A análise dos dados foi realizada de forma quantiqualitativa, baseada nos objetivos e na literatura estudada, utilizando-se a estatística descritiva, com apresentação de freqüências simples, porcentagem e interpretação das falas das participantes.

\section{APRESENTANDO E DISCUTINDO OS RESULTADOS}

Os resultados obtidos serão discutidos a partir de uma amostra de 109 mulheres atendidas no ambulatório da MEJC, com uma faixa etária entre 15 a 83 anos de idade. Quanto à escolaridade, $60,5 \%$ referiram o primeiro grau, e $18,3 \%$, o segundo; $66 \%$ eram casadas, e $17,4 \%$, solteiras; $79 \%$, católicas, $74 \%$ residiam em Natal, e $66 \%$ referiram ser do lar, 15,5\% eram autônomas, e 10\%, funcionárias públicas. No que concerne à paridade, $45 \%$ já tinham tido de duas a quatro gestações, e 14\% eram nulíparas. Quanto aos antecedentes familiares, as patologias mais citadas foram a hipertensão arterial (50\%), cardiopatias (42\%), diabetes (31\%) e câncer em $25 \%$. No que se refere à amamentação, $79 \%$ afirmaram essa conduta, e $21 \%$ referiram não terem amamentado seus filhos. Após essa caracterização, faremos uma discussão das questões relacionadas ao auto-exame de mama, objeto deste estudo.

Para análise desses dados, identificamos, primeiramente, que, das 109 mulheres entrevistadas, 82 $(75 \%)$ referiram se auto-examinar, enquanto $27(25 \%)$ nunca tinham realizado esse exame. Identificamos, ainda, que, desse contigente de mulheres, 17 (15,5\%) afirmaram não saber o significado de auto-exame de mama. Observamos, então, que, das 27 mulheres que nunca realizaram o auto-exame, 17 não sabem o seu significado.

Ao serem abordadas quanto ao conhecimento que tinham sobre o auto-exame de mama, reportaram-se com as seguintes colocações: (...) é o cuidado que a mulher tem com as medidas de prevenção em saúde (...) é o exame sistemático e periódico de palpação das mamas realizado pela mulher (...) é o exame da mama realizado pelo ginecologista.

As referências dessas mulheres quanto ao autoexame de mama são coerentes e respaldadas na literatura, com exceção das que citaram ser o ginecologista autor desse exame. Para a literatura, o auto-exame de mama é aquele realizado pela mulher, com a função importante de prevenção e promoção da doença, objetivando 
conhecimento detalhado da mulher sobre suas mamas, facilitando, assim, a percepção de qualquer alteração que possa ocorrer ${ }^{(11-13)}$.

Por meio da interação dos pesquisadores com as mulheres, durante as entrevistas, foi possível identificar as alterações encontradas nas mamas daquelas que se auto-examinavam. Das 82 (75\%) mulheres que realizavam o auto-exame, só 15 (18\%) detectaram alguma alteração assim relacionadas: 10 (12\%) referiram ter identificado nódulos em suas mamas; 3 (4\%) citaram os cistos, e 2 (2\%) observaram saída de líquido espesso dos mamilos.

Sabemos que a descoberta de alguma alteração na mama gera sentimentos negativos na mulher, principalmente o medo de um diagnóstico afirmativo de câncer. Essa realidade pode levar essa mulher a protelar sua consulta ao médico, pelo pavor dessa confirmação. Questionando-as, ainda, sobre que decisão tomaram frente à descoberta dessas alterações, todas (15) afirmaram terem procurado o médico para o devido tratamento. Esse fato é contrário ao que afirma a literatura, e as mulheres, por considerarem a mama um símbolo da feminilidade e da sexualidade, apresentam sentimentos de receio diante da possibilidade de câncer e cirurgias mutiladoras, quando detectam qualquer alteração mamária. Dessa forma, adiam a consulta ao especialista, provocando atrasos no diagnóstico precoce ${ }^{(18)}$.

As reações do medo em algumas mulheres, no entanto, podem ser motivo para a procura imediata do médico, e devem ser valorizadas como uma ação para o autocuidado que pode prevenir as conseqüências de um diagnóstico tardio, respaldando, assim, o comportamento das participantes deste estudo, diante das alterações identificadas, no momento do auto-exame, ao procurarem o médico.

Ao serem inquiridas sobre como realizavam o autoexame de mama, declararam os seguintes métodos utilizados por elas: (...) após a menstruação, palpo a mama em pé (...) deitada, passo óleo nas mamas, coloco um braço para trás e com o outro examino toda a mama (...) massageio as mamas quando estou deitada ou tomando banho, fazendo expressão nos mamilos (...) realizo a palpação em todos os quadrantes da mama e nas axilas, na posição deitada (...) faço a palpação das mamas com as pontas dos dedos com o outro braço para trás, sempre que tomo banho ou de frente para o espelho (...) faço a palpação quando estou deitada ou ensaboada no banho, colocando um braço para trás e com a outra mão palpo a mama em círculo e também as axilas.

Diante desses depoimentos, elas apresentam, até certo ponto, a compreensão e o significado de como se auto-examinar, pela construção que ocorre a partir da prática adquirida no cotidiano, pelas informações recebidas por meio das campanhas educativas e dos meios de comunicação, possibilitando a elas uma avaliação simples e tornando-as aptas a se conhecerem melhor.

Quando abordadas sobre o período mais indicado para realizar o auto-exame de mama, 47 (43\%) das 109 mulheres entrevistadas responderam que não sabem esse período; 39 (36\%) referiram o período pós-menstrual; 20 (18\%) relataram ser o pré-menstrual, e só 3 (3\%) afirmaram que o melhor momento seria o menstrual.

Segundo a literatura, o período mais favorável para o auto-exame de mama é o pós-menstrual, devido à menor influência hormonal, facilitando a palpação da mama e a detecção de nódulos, quando as mamas se apresentam mais flácidas e indolores ${ }^{(11-13)}$.

\section{CONSIDERANDO OS RESULTADOS}

Os resultados alcançados por meio dos dados desta pesquisa sobre o auto-exame de mama, em 109 mulheres de uma camada carente, deu-nos a possibilidade de identificar que a maioria (82) das entrevistadas referiram realizar o auto-exame no seu cotidiano, respaldadas pela literatura, quando estabeleceram conceitos, de certa forma corretos, sobre essa técnica. Entretanto, percebe-se a desinformação de 17 mulheres, sobre o tema, e a não realização do auto-exame de mama em 27 participantes.

A descoberta de alterações mamárias foi identificada por 15 mulheres ao se auto-examinarem, levando-as a uma decisão imediata quando resolveram procurar assistência médica para um diagnóstico correto e tratamento adequado.

No que se refere ao período favorável para a realização do auto-exame, predominou o desconhecimento de 47 mulheres sobre esse momento, conseqüentemente por falta de informações ou mesmo pelo desinteresse em saber o período correto.

No exercício da técnica de palpação da mama, as participantes do estudo nos fizeram compreender a importância da realização de um auto-exame com autoconfiança, possibilitando, assim, o exercício de uma prática saudável e preventiva a uma clientela de baixo nível socioeconômico e cultural.

Com esses resultados, entendemos a necessidade cada vez maior de fortalecimento dos programas de saúde direcionados à mulher, no que se 
refere ao conhecimento do próprio corpo, principalmente quanto ao auto-exame de mama, constituindo, dessa forma, subsídios para as reflexões do profissional de saúde sobre essa técnica, possibilitando um convite no redirecionamento de uma prática profissional mais humana, sob a forma de ensinar e assistir a mulher.

Finalmente, convém destacar a importância do

\section{REFERÊNCIAS BIBLIOGRÁFICAS}

1. Almeida AM, Mamede MV, Panobianco MS, Prado MAS, Clapis MJ. Construindo o significado da recorrência da doença: a experiência de mulheres com câncer de mama. Rev Latino-am Enfermagem 2001 setembro-outubro; 9(5): 63-9.

2. Freitas RJ, Conti RC. Quimioprevenção do câncer de mama. Já é chegada a hora? Femina 1993 novembro; 21 (11): 1181-4.

3. Fundação Oncocentro de São Paulo (BR). Câncer de mama. Controle no Estado de São Paulo: Manual de Orientação. São Paulo; 1994.

4. Barros ACSD, Nazário ACP. Fatores de risco para câncer de mama. In: Faria SL, Leme LHS, Alves FJ. O câncer de mama: diagnóstico e tratamento. Campinas (SP): Medsi; 1994. p.21-5.

5. Ministério da Saúde (BR). Campanha Nacional de Controle ao Câncer. Programa de Oncologia. Rio de Janeiro (RJ): Ministério da Saúde; 1990.

6. Pinotti JA, Barros ACSD. Anticoncepcionais orais e câncer de mama. Rev Ginecol Obstet 1992; 3(4):201-6.

7. Smeltzer SC, Bare BG. Avaliação e cuidados à paciente com distúrbios da mama. In: Brunner LS, Suddarth DS. Tratado de enfermagem médico-cirúrgica. Rio de Janeiro (RJ): Guanabara Koogan; 1994. p.1097-120.

8. Entrekin N. Câncer de mama. In: Clark JC, McGee RF. Enfermagem oncológica: um currículum básico. Porto Alegre (RS): Artes Médicas; 1997. p.401-15.

9. Koifman S. Incidência de câncer no Brasil. In: Minayo MCS, Organizadora. Os muitos Brasis: saúde e população na década de 80. São Paulo (SP): Hucitec; 1995. p.143-76.

10. Ministério da Saúde (BR). Instituto Nacional do Câncer. Coordenação de Programas de Controle de Câncer. A detecção do câncer de mama: atualização e recomendações. Rio de Janeiro (RJ): Ministério da Saúde; 1993.

11. Ministério da Saúde (BR). Instituto Nacional do Câncer. Coordenação de Programas de Controle de Câncer. Falando sobre doenças da mama. Rio de Janeiro (RJ): Ministério da Saúde; 1996.

12. Souen J. Detecção precoce de câncer de mama: experiência pessoal. Rev Bras Ginecol Obstet 1995 abril; 17(3):333-9.

13. Filipak RMA, Rodrigues MB, Back RVW, Carreño PAT, Urbanctz $A A$, Coelho $A$, et al. Câncer de mama: diagnóstico precoce. Femina 1993 fevereiro; 21(2):93-113.

14. Schraiber LB, Nemes MIB, Mendes GRB. Saúde do adulto: programas e ações na unidade básica. São Paulo (SP): Hucitec; 1996. autocuidado, tanto para as usuárias dos programas de saúde quanto para os profissionais que deles participam, tendo em vista a utilização de campanhas educativas com a inserção de novos conhecimentos, estimulando a participação da comunidade, facilitando o aprendizado, além de servir como estímulo à multiplicação de novos conhecimentos.

15. Ministério da Saúde (BR). Instituto Nacional do Câncer. Coordenação dos Programas de Controle do Câncer. Estimativas de incidência e mortalidade por câncer no Brasil para 1998. Rio de Janeiro (RJ): Ministério da Saúde; 1998. 16. Gomes JC. Diagnóstico clínico do câncer mamário. In: Faria SL, Leme LHS, Alves FJ. Câncer da mama: diagnóstico e tratamento. Campinas (SP): Medsi; 1994. p.29-38.

17. Ministério da Saúde (BR). Conselho Nacional de Saúde. Comissão Nacional de Ética em Pesquisas - CONEP Resolução $n^{\circ}$ 196/96. Dispõe sobre pesquisa envolvendo seres humanos. Rio de Janeiro (RJ): Ministério da Saúde; 1996.

18. Baruffi I. Tratado de oncologia toxicoginecológica e mamária. São Paulo (SP): Roca; 1985. 